\title{
Bio-plasmonics: Nano/micro structure of surface plasmon resonance devices for biomedicine
}

\author{
CHII-WANN LIN ${ }^{1,2,3, *}$, KUO-PING CHEN ${ }^{1}$, MING-CHII S U ${ }^{1}$, \\ CHIH-KUNG LEE AND CHIH-CHUNG YANG \\ ${ }^{1}$ Institute of Biomedical Engineering, National Taiwan University, No. 1, Sec. 4, Roosevelt Road, Taipei, \\ 106, Taiwan \\ ${ }^{2}$ Department of Electrical Engineering, National Taiwan University, No. 1, Sec. 4, Roosevelt Road, Taipei, \\ 106, Taiwan \\ ${ }^{3}$ Center for Nano Science and Technology, National Taiwan University, No. 1, Sec. 4, Roosevelt Road, \\ Taipei, 106, Taiwan \\ ${ }^{4}$ Institute of Applied Mechanics, National Taiwan University, No. 1, Sec. 4, Roosevelt Road, Taipei, 106, \\ Taiwan \\ ${ }^{5}$ Graduate Institute of Electro-Optical Engineering, National Taiwan University, No.1, Sec. 4, Roosevelt \\ Road, Taipei, 106, Taiwan \\ (*author for correspondence: E-mail: cwlinx@ntu.edu.tw)
}

Received 18 February 2005; accepted 15 September 2005

\begin{abstract}
We have reported a novel design of surface plasmon resonance (SPR) device, which uses alternating dielectric layers to enhance the SPR signal quality and modulate its resonant position. Admittance loci method of thin film design has been used in the design of such a device. The use of biomolecular thin film, such as DNAs or Porteins, in this design can result in resonant condition of wavelength changes as well. Bio-plasmonics is proposed for the research and development of novel devices, which use biomolecules as a part of the plasmon oscillation system to actively interact with nano/micro structure.
\end{abstract}

Key words: admittance loci, biosensor, surface plasmon resonance

\section{Introduction}

It is known that the free electrons of a metal can be treated as a high density liquid or plasma, which can then be excited by applied electromagnetic waves to have plasma oscillations. Such oscillations can exist in the bulk media and at the interface of a dielectric with a positive dielectric constant and a metal with a negative real part of the dielectric constant (Raether 1980, 1988). Such a phenomenon was first observed in metal grating in the early 1900's. Since then, its fundamental properties have been extensively studied in the vacuum by electron loss spectroscopy (Otto 1968; Kretschmann 1971). Fano was first pointed out conditions for the existence of evanescent surface waves, then Kretschmann realized the device by using a metallic-film-coated $(\sim 50 \mathrm{~nm})$ prism to generate a surface plasmon 
resonance (SPR) signal. SPR is the quanta representing of surface charge density oscillation in coherent fluctuation due to parallel polarized incident light. It has been found to be in good agreement with theoretical concepts and formulation of Maxwell theory (Salamon et al. 1997a). Since then, the Kretschmann prism-coupling device has been used extensively to study the optical properties of metallic thin films and later as a biosensor mainly due to two distinguishing features, i.e. high sensitivity and electric field enhancements. The unique characteristics of non-labeling and realtime monitoring can be used in many biochemical and biophysical applications (Homola et al. 1994, Salamon et al. 1997b). Meanwhile, novel devices, which are based on the use of dielectric materials and structural design, have been proposed to enhance the sensitivity and resolution of the device. These include long-range surface plasmon resonance (LRSPR) (Sarid 1981; Kou and Tamir 1987; Nenninger et al. 2001), coupled plasmon waveguide resonance (CPWR) (Salamon et al. 1997c), or near infrared light source (Nelson et al. 1999). Recently, we have reported a design based on dielectric mirror type of alternating dielectric multi-layer structure (Lin et al. in press). In this paper, we will discuss a general design method, admittance loci, which can be used for all these designs in details. Admittance loci method has been used in the optical thin film design for high performance optics and unconventional coatings in SPR device by Macleod (1992). It provides a systemic approach to the design of SPR devices, especial for the fine tuning of resonant angle or wavelength with prior knowledge of optical properties of biomolecules in the applications of biosesnor or biochip. Forward calculations by the Fresnel equation as mentioned in the most of SPR articles do provide theoretical prediction of the optical performance very well. Its computation has lead to the understanding of total reflectance and field distribution with specified device structure and materials. Admittance loci method provides a specific aim for SPR design, which is near zero reflectance with specified resonant angle under chosen wavelength or specified resonant wavelength [14]. With prior knowledge of optical properties of used materials, which include metals, dielectric, and biomolecules, we will be able to fine tune the resonant condition with multi-layer coatings, especially dielectric mirror type for high performance plasmonic devices. The other advantage comes from the batch fabrication of multi-layer optical coating on standard glass substrate, which is well established, and thus can provide lower cost SPR device for specific condition of measurement, comparing to use different wavelength of light source or higher refractive index of coupling prism.

We will discuss the method of admittance loci and then use different concentrations of single strand DNA to demonstrate the modulation of optical resonant angle. The use of biomolecular thin film in this design can result 
in resonant condition of wavelength changes as well. Bio-plasmonics is thus proposed for this kind of novel SPR devices, which use biomolecules as a part of the plasmon system to actively interact with nano/micro structure.

\section{Materials and methods}

\subsection{ADMITTANCE LOCI DESIGN METHOD}

As shown in the Fig. 1, as the incident light emitting from surrounding medium onto a multi-layer system, it can be treated as an equivalent admittance of $y_{\mathrm{e}}$. Admittance loci can be used to visualize the optical behavior of thin film, as we move a virtual reference plane from the substrate admittance $\left(y_{\text {sub }}\right)$ to the front surface of the multi-layer. The equivalent admittance, $y_{\mathrm{e}}$, beyond the reference plane will change due to the thin film thickness change. The plot of $y_{\mathrm{e}}$ on the complex plane gives trace of these changes, which is called admittance diagram or admittance loci. This method has been successfully applied for thin-film design of high-reflection (HR) and anti-reflection (AR) coatings in the past. However, its application for SPR sensor design has not been fully elucidated yet. We will discuss in details of the SPR sensor design in the following sections.

The refractive index of a light beam propagating on a medium is $N=c / v$, where constant $c$ is the velocity of light in a vacuum, and $v$ is the light speed in the material. This can also be expressed as $n-\mathrm{i} k$, where the real number $n$ is the refractive index, and the imaginary part $k$ is the extinction coefficient. For a multi-layer device, we first consider the reflectance of a thin film $\left(N_{1}\right)$ on a substrate $\left(N_{2}\right)$. The relationship of $E$ and $H$ at the boundaries of $a$ and $b$, as shown in Fig. 1 is

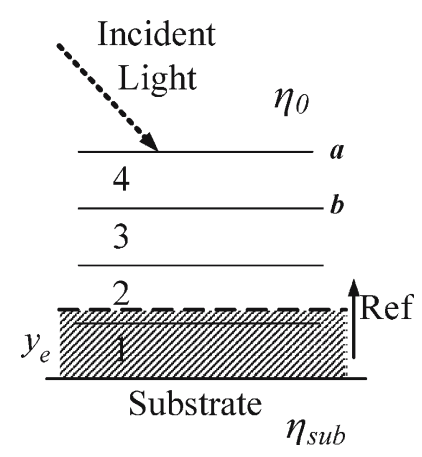

Fig. 1. Equivalent admittance of multi-layer over-coatings, where $\eta_{0}, \eta_{\text {sub }}$, and $\eta_{\mathrm{e}}$ is the admittance of the surrounding medium, substrate, and equivalent admittance, respectively, and treated as if moving a virtual reference plane (Ref) from the substrate admittance $\left(y_{\text {sub }}\right)$ to the front surface of the multi-layer. 


$$
\left\{\begin{array} { l } 
{ E _ { a 1 } { } ^ { + } = E _ { b 1 } { } ^ { + } \mathrm { e } ^ { \mathrm { i } \delta _ { 1 } } } \\
{ H _ { a 1 } { } ^ { + } = H _ { b 1 } { } ^ { + } \mathrm { e } ^ { \mathrm { i } \delta _ { 1 } } }
\end{array} \text { and } \left\{\begin{array}{l}
E_{a 1}{ }^{-}=E_{b 1}{ }^{-} \mathrm{e}^{-\mathrm{i} \delta_{1}} \\
H_{a 1}{ }^{-}=H_{b 1}{ }^{-} \mathrm{e}^{-\mathrm{i} \delta_{1}}
\end{array}\right.\right.
$$

The ' + ' sign means it is along the positive $z$-direction, the '-' sign designates the opposite direction, and $\delta_{1}$ is the phase factor due to thin film layer 1. The phase factor is a function of the refractive index, thickness, incident angle and wavelength. This is defined as

$$
\delta_{1}=2 \pi N_{1} d \cos \theta_{1} / \lambda .
$$

Therefore, Equation (1) can be rewritten either as

$$
\left\{\begin{array}{l}
E_{a}=E_{a 1}{ }^{+}+E_{a 1}{ }^{-}=E_{b} \cos \delta_{1}+\frac{\mathrm{i} H_{b}}{\eta_{1}} \sin \delta_{1}, \\
H_{a}=H_{a 1}{ }^{+}+E_{a 1}{ }^{-}=\mathrm{i} \eta_{1} E_{b} \sin \delta_{1}+H_{b} \cos \delta_{1} .
\end{array}\right.
$$

In matrix form,

$$
\left[\begin{array}{l}
E_{a} \\
H_{a}
\end{array}\right]=\left[\begin{array}{cc}
\cos \delta_{1} & \mathrm{i} \cdot \sin \delta_{1} / \eta_{1} \\
\mathrm{i} \cdot \eta_{1} \sin \delta_{1} & \cos \delta_{1}
\end{array}\right]\left[\begin{array}{c}
E_{b} \\
H_{b}
\end{array}\right] .
$$

The above $2 \times 2$ matrix is known as the characteristic matrix $\left(M_{1}\right)$ of the thin film. We define the equivalent admittance $\left(y_{\mathrm{e}}\right)$ as

$$
y_{\mathrm{e}}=H_{a} / E_{a} .
$$

Let $y_{\mathrm{e}}=C / B$. Equation (5) becomes

$$
\left[\begin{array}{l}
B \\
C
\end{array}\right]=\left[\begin{array}{cc}
\cos \delta_{1} & \mathrm{i} \cdot \sin \delta_{1} / \eta_{1} \\
\mathrm{i} \cdot \eta_{1} \sin \delta_{1} & \cos \delta_{1}
\end{array}\right]\left[\begin{array}{l}
1 \\
\eta_{\text {sub }}
\end{array}\right] .
$$

With this equivalent admittance, determining the reflectance of a multiple layer device on a substrate is similar to the single layer case. The characteristic matrix can likewise be used to obtain an equivalent admittance and reflectance:

$$
\left[\begin{array}{c}
E_{a} \\
H_{a}
\end{array}\right] \Rightarrow\left[\begin{array}{l}
B \\
C
\end{array}\right]=\prod_{j=1}^{a}\left[\begin{array}{cc}
\cos \delta_{j} & \mathrm{i} \cdot \sin \delta_{j} / \eta_{j} \\
\mathrm{i} \cdot \eta_{j} \sin \delta_{j} & \cos \delta_{j}
\end{array}\right]\left[\begin{array}{l}
1 \\
\eta_{\mathrm{sub}}
\end{array}\right] .
$$

The reflectance can then be written as

$$
R=\left(\frac{\eta_{0}-y_{\mathrm{e}}}{\eta_{0}+y_{\mathrm{e}}}\right)^{2} \text {. }
$$

Other than calculating the reflectance by using Equation (8), we can also employ this approach to design a multi-layer device. We will begin with a 
single layer of dielectric thin film $(y)$ and the equivalent admittance $\left(y_{\mathrm{e}}\right)$ with fixed wavelength and incident angle. One layer structure is $y_{0}|y| y_{\text {sub }}$, and $\delta$ means the phase thickness. The equivalent admittance is:

$$
y_{\mathrm{e}}=\alpha+\mathrm{i} \beta=C / B=\frac{y_{\text {sub }} \cos \delta+\mathrm{i} y \sin \delta}{\cos \delta+\mathrm{i}_{\text {sub }} / y \sin \delta} .
$$

Making real and imaginary parts on both sides of Equation (9) equal, we can get:

$$
\begin{aligned}
& \left\{\begin{array}{c}
\alpha \cos \delta-\frac{y_{\text {sub }}}{y} \beta \sin \delta=y_{\text {sub }} \cos \delta \\
\beta \cos \delta+\frac{y_{\text {sub }}}{y} \alpha \sin \delta=y \sin \delta
\end{array}\right. \\
& {\left[\begin{array}{cc}
\alpha-y_{\text {sub }} & -\frac{y_{\text {sub }}}{y} \beta \\
\beta & \alpha \frac{y_{\text {sub }}}{y}-y
\end{array}\right] \cdot\left[\begin{array}{c}
\cos \delta \\
\sin \delta
\end{array}\right]=0 .}
\end{aligned}
$$

For non-trivial solution of $\cos \delta, \sin \delta$, we can have the solutions as follows.

$$
\begin{aligned}
& \left|\begin{array}{cc}
\alpha-y_{\text {sub }} & -\frac{y_{\text {sub }}}{y} \beta \\
\beta & \alpha \frac{y_{\text {sub }}}{y}-y
\end{array}\right|=0 \Rightarrow\left(\alpha-y_{\text {sub }}\right)\left(\alpha \frac{y_{\text {sub }}}{y}-y\right)+\frac{y_{\text {sub }}}{y} \beta^{2}=0, \\
& \left(\alpha-\frac{1}{2}\left(y_{\text {sub }}+\frac{y^{2}}{y_{\text {sub }}}\right)\right)^{2}+\beta^{2}=\frac{1}{4}\left(y_{\text {sub }}-\frac{y^{2}}{y_{\text {sub }}}\right)^{2} .
\end{aligned}
$$

For a dielectric layer of admittance, it is a circle starting from $\left(y_{\text {sub }}, 0\right)$ with clockwise spiral due to the changes of film thickness and cross the real axis again at $\left(y^{2} / y_{\text {sub }}, 0\right)$ for every quarter-wavelength thickness as indicated by the Equation (13). The starting point can be anywhere on the circular locus. It will simply result in a different circle that will be centered on the real axis and cut it in two points $\alpha$ and $\beta$ such that $\alpha \beta=y^{2}$. One can then plot for isoreflectance contours, isophase contours, or electric-field contours for different purposes of visualization. This method will allow us to calculate the reflectance with the oblique incident angle (as in conventional SPR design), or with arbitrary structure (as in the multi-layer design).

Figure 2(a) shows an example locus of $\mathrm{TiO}_{2}$ thin film $(n=2.27)$ with thickness from 0 to $80 \mathrm{~nm}$ on top of a glass substrate $(n=1.5)$ in the air. Figure 2(b) shows an example of anti-reflectance coating under normal incident of light $(\lambda=633 \mathrm{~nm})$ on a common glass $(n=1.5)$.

We will now discuss the special case of total internal reflectance (TIR). TIR happens as the incident angle is larger than critical angle when light incident from dense medium to rare medium. 

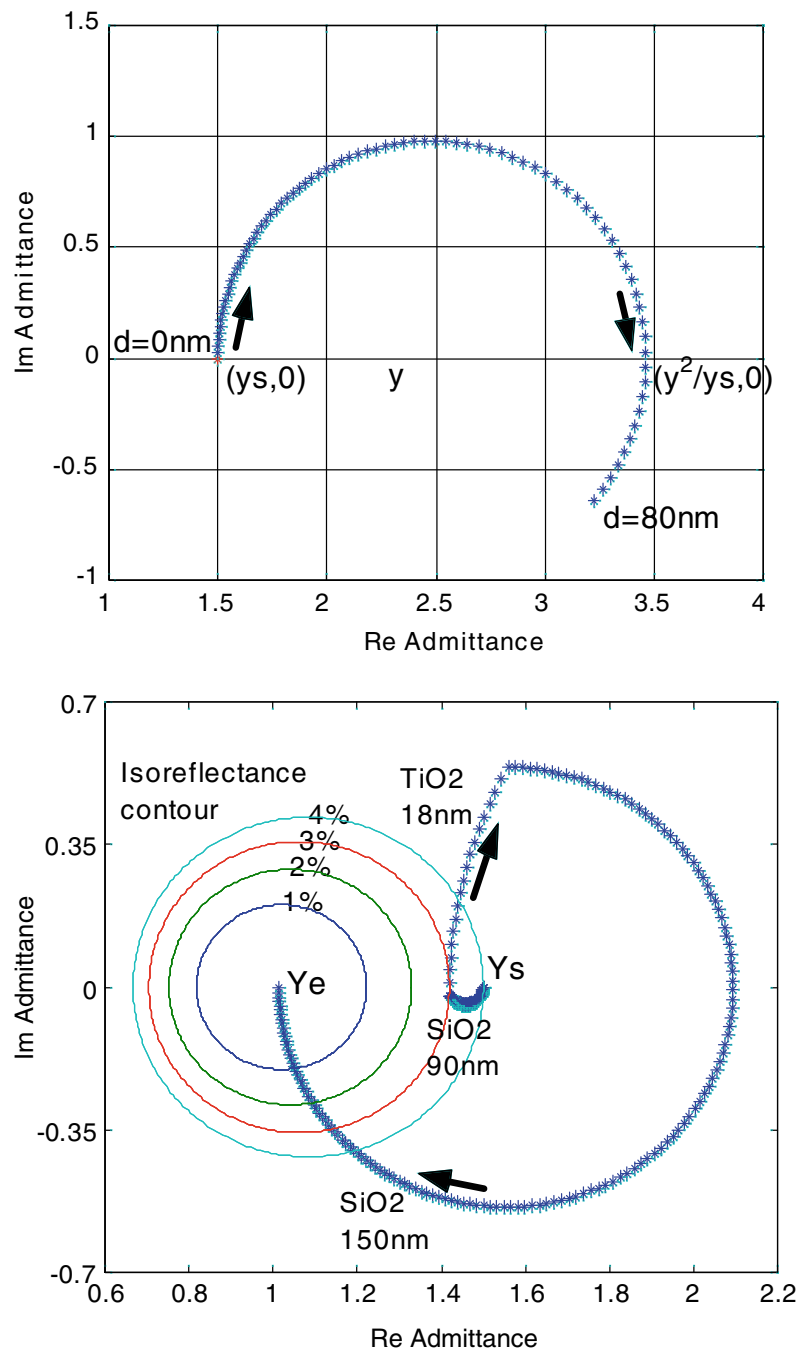

Fig. 2. Examples of admittance loci design. (a) Admittance locus of $80 \mathrm{~nm}$ layer of $\mathrm{TiO}_{2}$ on top of glass substrate. The clockwise trace will start from substrate $\left(y_{\text {sub }}, 0\right)$ and follow the equation passing through $\left(y^{2} / y_{\text {sub }}, 0\right)$ then end at its designed thickness. (b) A multi-layer device with $\mathrm{SiO}_{2}(90 \mathrm{~nm}), \mathrm{TiO}_{2}(18 \mathrm{~nm})$, and $\mathrm{SiO}_{2}(150 \mathrm{~nm})$ can result in zero reflectance as shown in an isoreflectance plot, with $\mathrm{TiO}_{2}(n=2.279)$ and $\mathrm{SiO}_{2}(n=1.4517)$.

As shown in the Fig. 3, when $\theta_{0}>\theta_{\mathrm{c}} \Rightarrow \sin \theta_{1}>1, \theta_{1}$ is a complex number, and making

$$
\begin{aligned}
\eta_{1 \mathrm{~s}}=n_{1} \cos \theta_{1}=n_{1}\left(1-\sin ^{2} \theta_{1}\right)^{\frac{1}{2}} & =n_{1}\left(1-\left(n_{0} \sin \theta_{0} / n_{1}\right)^{2}\right)^{\frac{1}{2}} \\
& =\left(\mathrm{n}_{1}{ }^{2}-n_{0}{ }^{2} \sin ^{2} \theta_{0}\right)^{\frac{1}{2}} .
\end{aligned}
$$



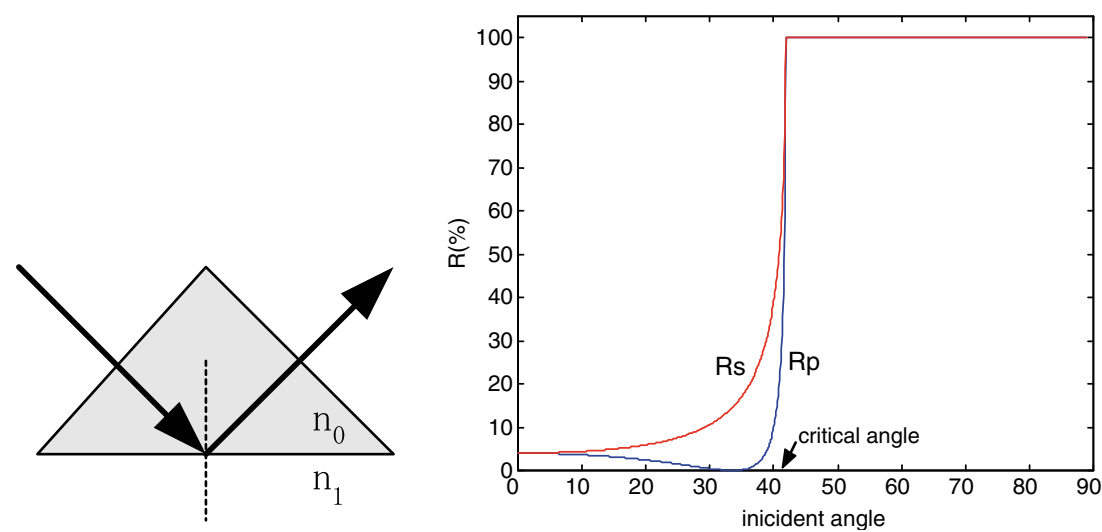

Fig. 3. Reflectance at the prism $(n=1.5)$-air interface due to s and p-polarization of incident light.

The result of the TIR admittance is a pure imaginary number, negative for s-polarization and positive for p-polarization. By the correction of oblique incident, the admittance of $\mathrm{p}$ and s polarization can be written as follows.

$$
\left\{\begin{array}{l}
\eta_{\mathrm{s}}=-\mathrm{i}\left(n_{0}^{2} \sin ^{2} \theta_{0}-n^{2}\right)^{\frac{1}{2}} / \cos \theta_{0}=-\mathrm{i} S \\
\eta_{\mathrm{p}}=n^{2} / \eta_{\mathrm{s}}=+\mathrm{i} P
\end{array}\right.
$$

The admittance is a real number when incident angle is below than critical angle but a pure imaginary number beyond the critical angle. For p-wave, the admittance locus will start at real axis and approach to $\infty$, as the incident angle becoming larger. Beyond the critical angle, the admittance will go to the positive imaginary axis and approaching zero with reflectance being $100 \%$. And s-wave goes in the other way from real axis to negative imaginary axis passing by zero.

For the special case of SPR, we will examine the admittance loci about ideal metal with absorbance $(y=-\mathrm{ik})$, as in a Kretschmann type SPR device (the prism is coated with a single high-performance metal layer). We are treating glass prism as the incident medium and the surrounding air as the substrate. Thus the starting admittance for the film is on the imaginary axis, negative part for s-polarization and the positive part for p-polarization.

It will show a near zero reflectance at a particular angle of incident and metal thickness as shown in the Fig. 4(a). The condition is very sensitive to angle of incidence. This very narrow drop in reflectance to a very low value is a surface plasmon wave on the metal film. 
(a)

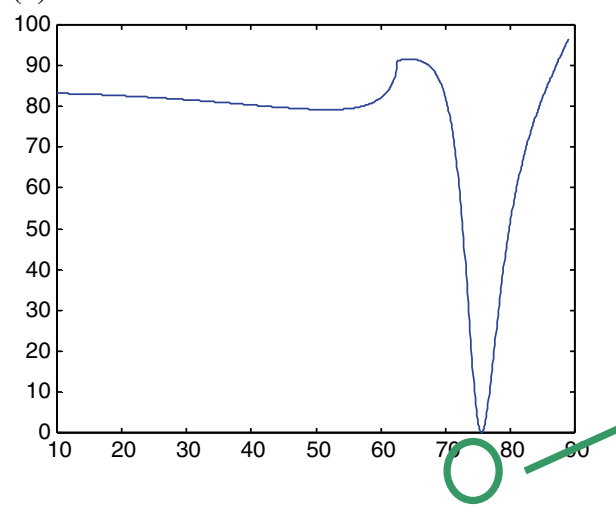

(b)

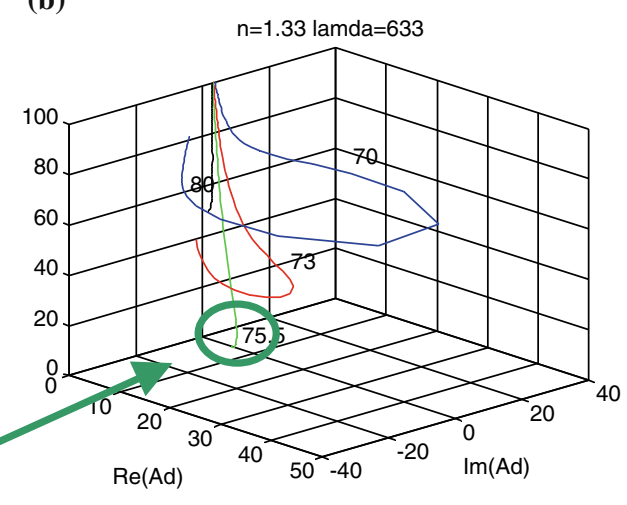

Fig. 4. (a) It shows an example case of SPR with prism (dense medium) $n=1.5$, gold film: $d=50 \mathrm{~nm}$, less dense medium: water $n=1.33$, and light source at $633 \mathrm{~nm}$. The critical angle is $62^{\circ}$ and the reflectance dip at $74.5^{\circ}$, which is SPR angle. (b) The combination of the admittance loci and reflectance for different incident angles.

The phase thickness will become a pure imaginary number under such circumstance and shown as follows:

$$
\begin{gathered}
\delta=\frac{2 \pi}{\lambda}(-\mathrm{ik}) d=-\mathrm{i} \gamma, \\
\left\{\begin{array}{c}
\cos \delta=\cosh \gamma, \\
\sin \delta=-\mathrm{isinh} \gamma
\end{array}\right.
\end{gathered}
$$

Characteristic matrix of the film will become,

$$
\left[\begin{array}{l}
B \\
C
\end{array}\right]=\left[\begin{array}{cc}
\cosh \gamma & \frac{\mathrm{i}}{k} \sinh \gamma \\
-\mathrm{iksinh} \gamma & \cosh \gamma
\end{array}\right]\left[\begin{array}{c}
1 \\
y_{\text {sub }}
\end{array}\right]
$$

Because $y_{\mathrm{e}}=C / B=\alpha+\mathrm{i} \beta$, the relation of real and imaginary parts of admittance loci is,

$$
\left\{\begin{array}{l}
\left(\alpha-y_{\mathrm{sub}}\right) \cosh \gamma=\frac{\beta}{k} y_{\mathrm{sub}} \sinh \gamma, \\
\beta \cosh \gamma=-\left(k+\frac{\alpha}{k} y_{\mathrm{sub}}\right) \sinh \gamma
\end{array}\right.
$$

for non-trivial solution of $\gamma$, we can get the locus of $\alpha$ and $\beta$,

$$
\alpha^{2}-2 \frac{y_{\mathrm{sub}}^{2}-k^{2}}{2 y_{\mathrm{sub}}} \alpha+\beta^{2}=k^{2} .
$$


It is also a circle locus with center on $\left(\frac{y_{\text {sub }}^{2}-k^{2}}{2 y_{\text {sub }}}, 0\right)$ and radius of $\frac{y_{\text {sub }}^{2}+k^{2}}{2 y_{\text {sub }}}$. It goes from $(0, k)$ to $(0,-k)$. The different $y_{\text {sub }}$ and $k$ will change the radius and center of the locus.

From above, we know that if we put a dielectric thin film on top of the thin metal film, provided the added thin film has no losses, then the admittance of the film-substrate combination must remain on the imaginary axis and returning to starting point every half-wave thickness. Given that the starting point is on the axis, the only way in which the admittance can be made to leave it is by an absorbing layer. A thin layer is deposited over the metal next to the surrounding air. The starting admittance is on the imaginary axis, but the dielectric layer modifies that position, so that the starting point for the metal locus is changed. The combination of negative imaginary phase thickness and positive imaginary admittance inverts the way in which the circles are described. Because the metal loci on the imaginary axis are clustered closely together, almost intersecting, a small change in starting point produces an enormous change in the locus, and hence in the point at which it cuts the real axis, leading to a substantial change in reflectance. Under appropriate incident angle and thickness of a metal film, it can have near zero reflectance. Provided that the film is very thin, then an additional tilt of the system will be sufficient to pull the intersection of the metal locus with the real axis back to the incident admittance, and so the effect can be interpreted as a shift in the response rather than a damping.

By the theorem of the thin film analysis as mentioned in the above sections, we can simulate the SPR phenomenon by the characteristic matrix and admittance loci. The calculation has been implemented by MATLAB 5.3 (Mathwork Inc.) for the design of SPR thin film to modulate the resonance angle and wavelength as well.

\subsection{MATERIALS AND FABRICATION}

We used SuperFrost-100 (MENZEL-GLASER, German, refractive index = 1.51), a standard microscopy glass slide, as the substrate for metallic thin film deposition. The metallic thin film used was gold $(\mathrm{Au})$, the high/low refractive index materials used were $\mathrm{TiO}_{2}(n=2.28)$ and $\mathrm{SiO}_{2}(n=1.46)$, with $\mathrm{Cr}$ for the surface layer. All deposited materials $\left(\mathrm{SiO}_{2}, \mathrm{TiO}_{2}, \mathrm{Cr}, \mathrm{Au}\right)$ used had purity $>99.99 \%$. The glass slides were cleansed with piranha solution $\left(\mathrm{H}_{2} \mathrm{SO}_{4}: \mathrm{H}_{2} \mathrm{O}_{2} 3: 1\right)$ at $90^{\circ} \mathrm{C}$ to remove organic debris. The surface was then immersed in an ultrasonic water bath for $10 \mathrm{~min}$, and cleansed with detergent and acetone. Finally, ethanol and cotton paper were used to thoroughly drain the water. The sequence of deposition for the $\mathrm{Au}$ film was $1 \mathrm{~nm}$ of $\mathrm{Cr}$, followed by $50 \mathrm{~nm}$ of $\mathrm{Au}$ at a pressure of $\sim 1.6^{*} 10^{-5}$ Torr, 
in an e-beam evaporator $(9 \mathrm{KV})$. The deposition process was performed at the Precision Instrument Development Center in Hsinchu, Taiwan at $0.1-0.2 \mathrm{~nm} / \mathrm{s}$. The thickness of the deposited thin film was monitored using a quartz crystal microbalance (QCM), which was carefully calibrated to the different characteristics of deposited materials. To improve the quality of the thin films and its adherence, the substrate was heated at various temperature during the evaporation of the gold layer $\left(100^{\circ} \mathrm{C}\right)$ and the dielectric layers $\left(300^{\circ} \mathrm{C}\right)$. It is generally known that better results can be obtained when $\mathrm{Au}$ and $\mathrm{TiO}_{2}$ are pre-melted. Hence, this is an essential process in making multi-layered devices.

\subsection{VERIFICATION}

Our multi-layer SPR device was tested on Cary 50 (Varian Inc., USA), a spectrophotometer for spectral measurements in a transmittance mode; and EP3 (Nanofilm Inc. Germany), an imaging ellipsometer for optical properties of thin film and SPR spectra.

\subsection{BIOLOGICAL SAMPLES}

Escherichia coli plasmid DNA are purified and diluted to different concentrations. The original concentration is $935 \mu \mathrm{g} / \mathrm{ml}$. Physical adsorption method is used to immobilize the DNA on the gold film by loading $5 \mu \mathrm{l}$ DNA solution on the gold spot (each spot diameter is $4 \mathrm{~mm}$ ) as shown in the Fig. 5. The sample is then put in a oven with temperature setting on $60^{\circ} \mathrm{C}$ for $30 \mathrm{~min}$. After evaporation of water, the DNA is immobilized on the gold surface as a DNA thin film. The sample is then measured by the EP3 and the range of the incident angle is $40-49^{\circ}$.

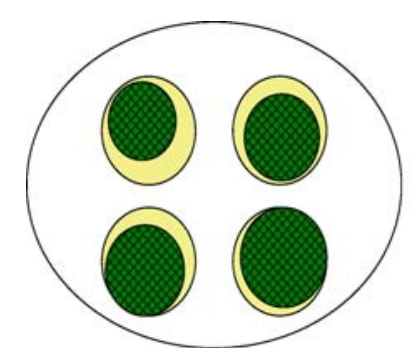

Fig. 5. Various dilutions of DNA spots arrangement on the SPR array. 

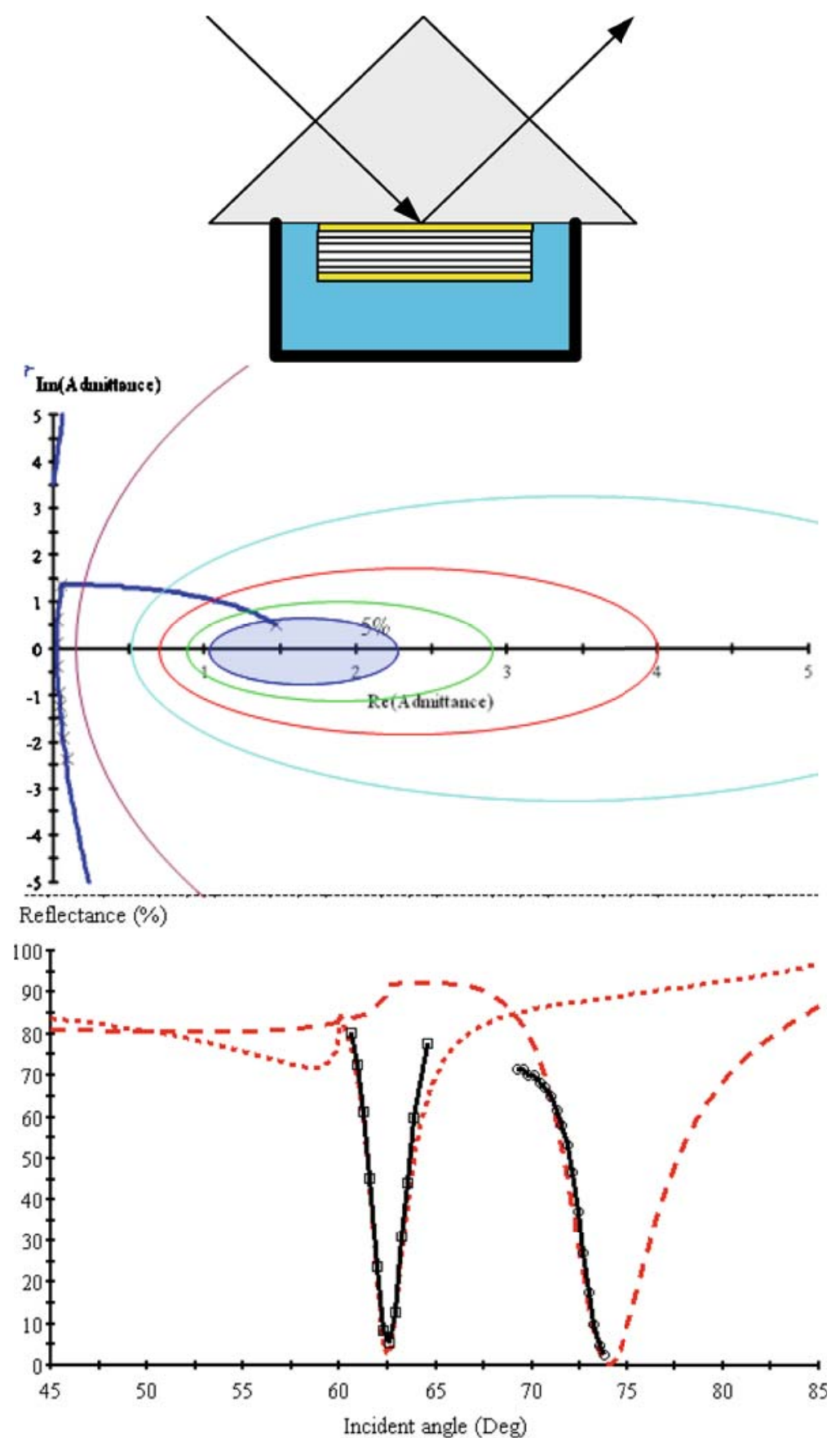

Fig. 6. As in the SPR configuration (a) the symmetric SPR device with DBR overlay structure (b) the admittance loci will leave the imaginary axis and could have the chance to approach the real axis to let the reflectance becomes zero, and (c) the resultant SPR spectrum (solid line) shows improved characteristics of smaller resonant angle and FWHM with $633 \mathrm{~nm}$ incident light under water. 


\section{Results and discussion}

We have reported the uses of alternating dielectric layers of high/low refractive index materials $\left(\mathrm{Au} /\left(\mathrm{SiO}_{2}, / \mathrm{TiO}_{2}\right)_{y} / \mathrm{Au}(y=4)\right)$ to modulate SPR properties for the sensing of biochemical solutions, as shown in the Fig. 6(a) [12]. With admittance loci method, we can directly visualize the effects of overlay coatings as shown in the Fig. 6(b). The resultant SPR spectrum (solid line in the Fig. 6(c)) of this new device exhibited smaller resonant angle and full width half maximum (FWHM). It also results in improved characteristics of dynamic range and sensitivity. In this paper, we gave detail derivations of admittance loci method and the interactions of this multi-layer structure with DNA and protein biomolecules.

With the design method and fabrication processes of such a multi-layer SPR devices, we will be able to investigate the bioplasmonics by treating the biomolecular layer as an equivalent optical thin film and design for a specific resonant condition. Figure 7 shows another aspect of wavelength modulation by multi-layer device. With the same structure design in our previous paper, it results in the shifting of resonant wavelength from $1020 \mathrm{~nm}$ (dashed line) to $633 \mathrm{~nm}$ (solid line), which will allow directed visual examination of biomolecular interactions.

The feasibility of bioplasmonics can be demonstrated by using dry samples of immobilized DNA molecules. E. coli plasmid DNA are purified and diluted to different concentrations. The original concentration is $935 \mu \mathrm{g} / \mathrm{ml}$. Physical adsorption method is used to immobilize the DNA on the gold film by loading $5 \mu \mathrm{l}$ DNA solution on the gold spot (each spot diameter is $4 \mathrm{~mm}$ ). The sample is put in a oven with temperature setting on $60^{\circ} \mathrm{C}$ for 30 min. After evaporation of water, the DNA is immobilized on the gold

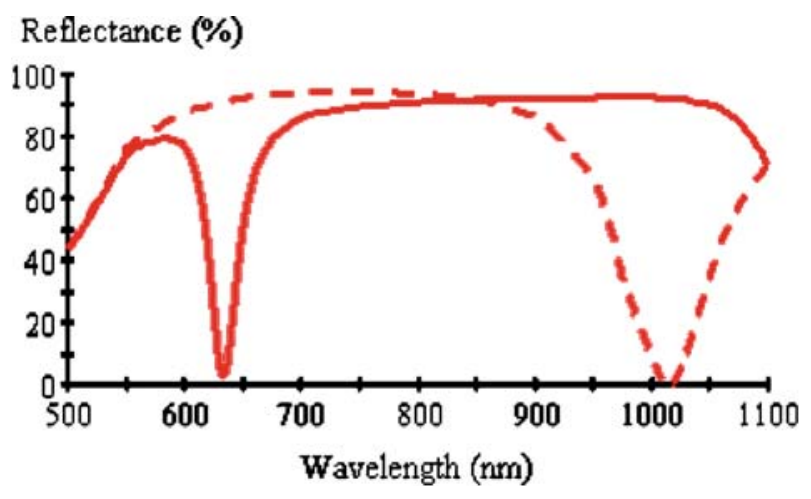

Fig. 7. The effect of multi-layer coatings on the modulation of SPR resonant wavelength results in the shifting from $1020 \mathrm{~nm}$ (dashed line) to $633 \mathrm{~nm}$ (solid line), which will allow directed visual examination of biomolecular interactions. 


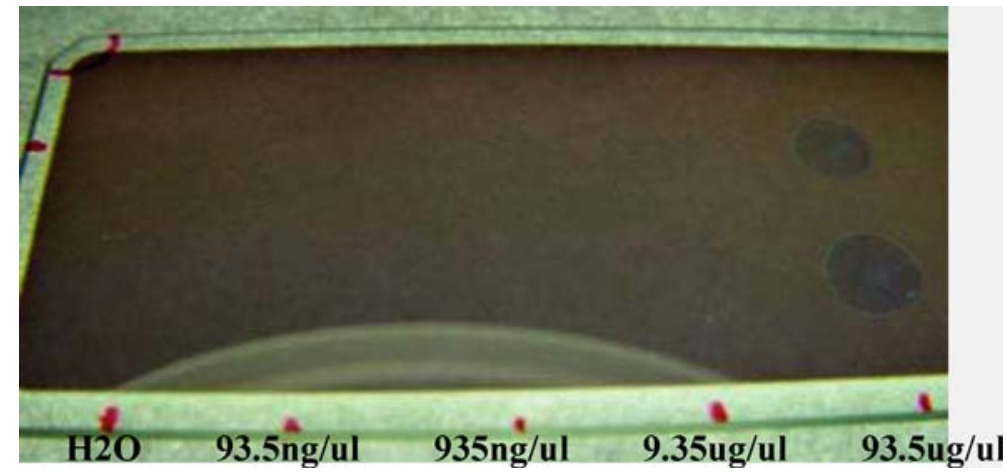

Fig. 8. The application of various concentration of DNA on multi-layer SPR device can result in color changes.

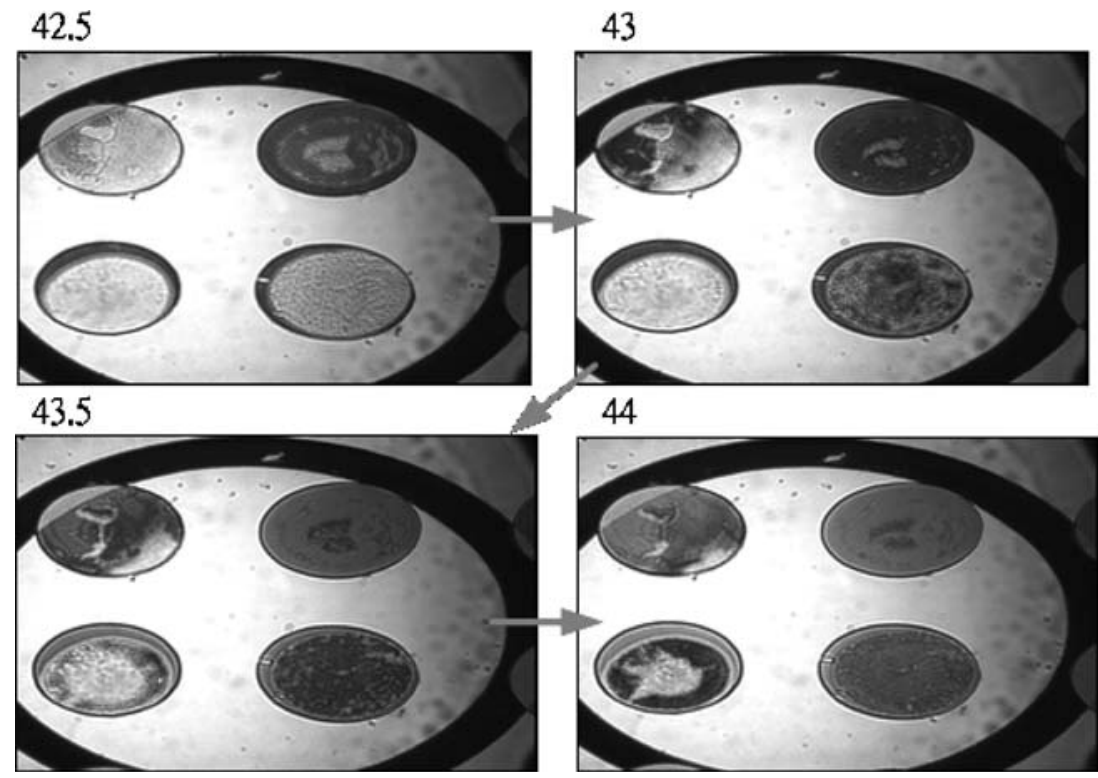

Fig. 9. SPR images of DNA samples with incident angle from $42.5^{\circ}-44^{\circ}$.

surface as a DNA thin film. On the $93.5 \mu \mathrm{g} / \mathrm{ml}$ spot, we can clearly identify the color change as shown in the Fig. 8. It is possibly due to interference effect between biomolecular thin film and overlay coatings, which results in both transmitted and reflective color changes. Such a color change can be a very high sensitive optical sensor by further optimization of the optical thin film design, such as bistable optical coatings in the future.

By measuring SPR intensity and angle shift, the adsorption condition and thickness can be characterized for higher sensitivity. The sample is measured by the EP3 and the range of the incident angle is $40-49^{\circ}$. The 
Table 1. The resonance angle of the different concentration DNA spots

\begin{tabular}{llccll}
\hline & Pure gold & $10000 \times$ & $1000 \times$ & $100 \times$ & $10 \times$ \\
\hline SPR angle & 42.7 & 42.85 & 43.02 & 43.49 & 43.95 \\
Thickness $(\mathrm{nm})$ & - & 3.2 & 7 & 16 & 24 \\
\hline
\end{tabular}

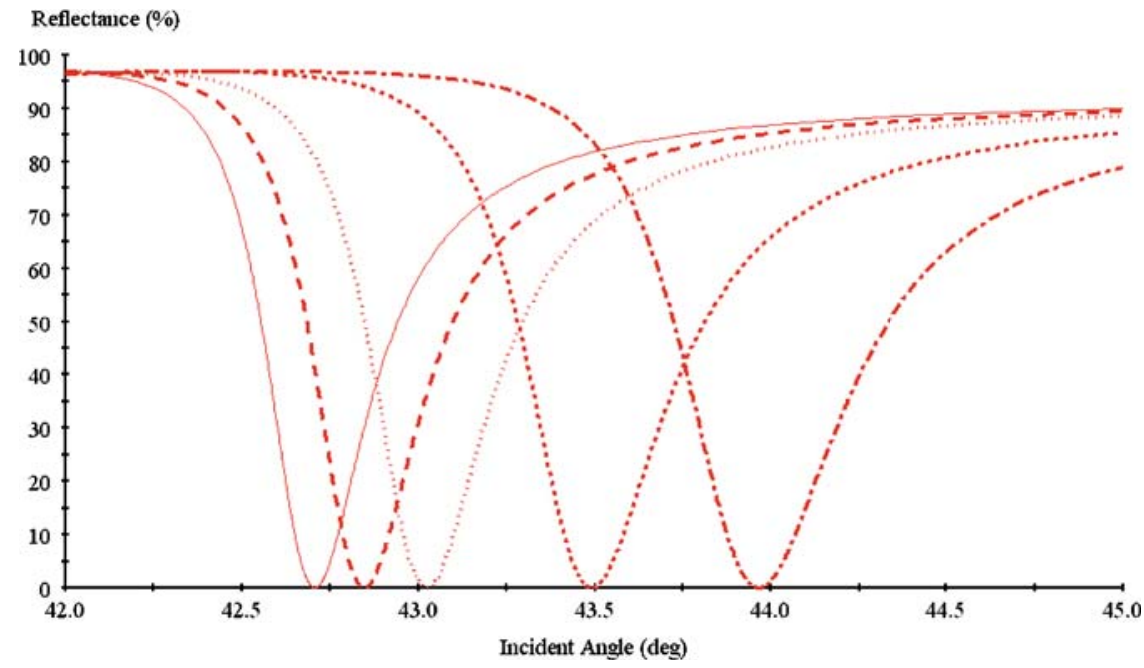

Fig. 10. Reflectance spectra of the different dilution of DNA samples in the SPR images.

results of SPR images are shown in the Fig. 9 with four incident angles. In the image, the bright region is due to TIR of glass/Au/air with highreflectance exceeding the critical angle. The dark region on the gold film is because of the surface plasmon resonance, which causes the diminishing of reflectance. At certain angle near SPR, the same intensity region means the same phase thickness of DNA. As the organic thin film becomes thicker, the resonance angle also becomes larger as shown in the Table 1.

We can plot the R- $\theta$ curves of the different regions in the SPR images. As shown in the fitted spectra of Fig. 10, different regions on the same image will have different reflectance as a function of the incident angle. This result can be used to calculate the thickness of the DNA thin film with refractive index value of DNA $(n=1.3386)$. The estimated thickness of different DNA concentrations will be $24,16,7$, and $3.2 \mathrm{~nm}$, respectively as shown in Table 1.

\section{Conclusion}

We have reported a novel design of SPR device, which uses alternating dielectric layers to enhance the SPR signal quality and modulate its 
resonant position. In this paper, not only we gave detail derivations of admittance loci method of device design but also showed the interactions of DNA molecules can result in significant resonant wavelength change or color change, which is possibly due to equivalent thickness changes during immobilization. Bio-plasmonics, which use biomolecules as a part of the plasmon oscillating system, can be designed by using admittance loci method for specific resonant conditions. The treatment of effective medium theory for biomolecules would thus require further investigations to have more accurate results for medical device applications.

\section{Acknowledgements}

This project is supported by National Science and Technology Program in Pharmaceuticals and Biotechnology, National Science Council, Taiwan, R.O.C., NSC 93-2323-B002-001 and NSC 93-2323-B002-004.

\section{References}

Homola, J., S.S Yee, and G. Gauglitz. Sensors Actuators B 54 3, 1994.

Kou, F.Y. and T. Tamir. Optics Lett. 12 367, 1987.

Kretschmann, E. Z. Phys. 241 313, 1971.

Lin, C.-W., K.-P. Chen, C.-K. Lee and S.-M. Lin. Sensors Actuators B in press. In: Macleod, H.A. (ed.)

Tutorials in Optics. Optical Society of America, Washington, DC., p. 121, 1992.

Nelson, B.P., A.G. Frutos, J.M. Brockman and R.M. Corn. Anal Chem. 71 3928, 1999

Nenninger, G.G., P. Tobiska, J. Homola and S.S. Yee. Sensors Actuators B 74 145, 2001.

Otto, A. Z. Phys. 216 398, 1968.

Raether, H. Excitation of Plasmons and Interband Transitions by Electrons, Springer-Verlag, Berlin, 1980.

Raether, H. Surface Plasmons on Smooth and Rough Surfaces and on Gratings, ch. 2. Springer-Verlag, Berlin, 1988.

Salamon, Z., H.A. Macleod and G. Tollin. Biochim. Biophys. Acta 1331 117, 1997a.

Salamon, Z., H.A. Macleod and G. Tollin. Biochim. Biophys. Acta 1331 131, 1997b.

Salamon, Z., H.A. Macleod and G. Tollin. Biophys. J. 73 2791, 1997c.

Sarid, D. Phys. Rev. Lett. 47 1927, 1981 